International Journal of Pure and Applied Mathematics

Volume 92 No. 4 2014, 569-578

ISSN: 1311-8080 (printed version); ISSN: 1314-3395 (on-line version)

url: http://www.ijpam.eu

doi: http://dx.doi.org/10.12732/ijpam.v92i4.11

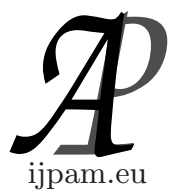

\title{
POSTULATION OF GENERAL UNIONS OF DECORATED LINES
}

\author{
E. Ballico \\ Department of Mathematics \\ University of Trento \\ 38123 Povo (Trento) - Via Sommarive, 14, ITALY
}

\begin{abstract}
For all integers $a \geq 0$ and $r \geq 3$ an $a$-decorated line $D \subset \mathbb{P}^{r}$ is a scheme union of a line $D_{\text {red }}$ and $a$ tangent vectors of $\mathbb{P}^{r}$ at points of $D_{\text {red }}$. Here we study the postulation of general disjoint unions of $a$-decorated lines.
\end{abstract}

AMS Subject Classification: 14N05, 14H99

Key Words: postulation, Hilbert function, unions of lines, decorated lines

\section{Introduction}

Fix a line $L \subset \mathbb{P}^{r}, r \geq 2$, an integer $a \geq 0$ and a finite set $S \subset L$ such that $\sharp(S)=a$. For each $P \in S$ fix a degree two zero-dimensional scheme $v_{P} \subset \mathbb{P}^{r}$ such that $\operatorname{deg}\left(v_{P}\right)=2$ and $\left\langle v_{P}\right\rangle \neq L$, where \langle\rangle denote the linear span; we call $v_{P}$ a tangent vector with $P$ as its reduction and the line $\left\langle v_{P}\right\rangle$ as its support, or the tangent vector of $\left\langle v_{P}\right\rangle$ at $P$. Let $X \subset \mathbb{P}^{r}$ be the minimal closed subscheme containing $L$ and each $v_{P}, P \in S$. We say that $X$ is an $a$-decorated line with $L$ as its support, $S$ as the support of its nilradical (or of the nilradical of the sheaf $\left.\mathcal{O}_{X}\right)$ and the tangent vectors $\left\{v_{P}\right\}_{P \in S}$ as its nil-directions. The scheme $X$ has dimension $1, X_{\text {red }}=L, h^{0}\left(\mathcal{O}_{X}\right)=a+1, h^{1}\left(\mathcal{O}_{X}\right)=1+a$. By construction the line bundle $\mathcal{O}_{X}(1)$ is very ample and $x+a+1$ is the Hilbert polynomial $p_{X}(x)$ of $X$ with respect to $\mathcal{O}_{X}(1)$. We have $h^{1}\left(\mathcal{O}_{X}(x)\right)=0$ for all $x \geq 0$ and hence $h^{0}\left(\mathcal{O}_{X}(x)\right)=x+1+a$ for all $x \geq 0$. It is elementary to check that $h^{1}\left(\mathcal{I}_{X}(t)\right)=0$ for all $t \geq a-1$. For all integers $r \geq 3, t>0$ and $a \geq 0$

Received: January 19, 2014

(c) 2014 Academic Publications, Ltd. url: www.acadpubl.eu 
let $W(r, t, a)$ be the set of all disjoint unions $X \subset \mathbb{P}^{r}$ of $t a$-decorated lines of $\mathbb{P}^{r}$. The set of all $a$-decorated lines of $\mathbb{P}^{r}$ with a fixed line $D$ as their support is parametrized by an irreducible variety of dimension $a+r-1$. Hence $W(r, t, a)$ is parametrized by an irreducible variety. Hence (as in [14] for the case $a=0$ ) it makes sense to compute the Hilbert function of a general element of $W(r, t, a)$. In this paper we prove the following result

Theorem 1. Fix integers $r, k, a, t$ such that $r \geq 3, t>0, k>0$ and $0 \leq$ $a \leq k+1$. Let $X$ be a general element of $W(r, t, a)$. Then either $h^{0}\left(\mathcal{I}_{X}(k)\right)=0$ or $h^{1}\left(\mathcal{I}_{X}(k)\right)=0$.

We work over an algebraically closed field $\mathbb{K}$ such that either char $(\mathbb{K})=0$ or $\operatorname{char}(\mathbb{K})>k$ (see Remark ++ for the positive caracteristic case).

\section{Preliminaries}

For all integers $r \geq 3, k>0$ and $a \geq 0$. Set $x_{r, k, a}:=\left\lfloor\left(\begin{array}{c}r+k \\ r\end{array}\right) /(k+1+a)\right\rfloor$ and $y_{r, y, a}:=\left(\begin{array}{c}r+k \\ r\end{array}\right)-(k+1+a) x_{r, k, a}$. We have $0 \leq y_{r, k, a}$ and

$$
(k+1+a) x_{r, k, a}+y_{r, k, a}=\left(\begin{array}{c}
r+k \\
r
\end{array}\right)
$$

Remark 1. Fix integers $r, k, a$ such that $r \geq 3, k>0$ and $0 \leq a \leq k+1$. To prove Theorem 1 for all quadruples $(r, k, a, t)$ is sufficient to prove that $h^{1}\left(\mathcal{I}_{X}(k)\right)=0$ for some $X \in W\left(r, x_{r, t, a}, a\right)$ and that $h^{0}\left(\mathcal{I}_{Y}(k)\right)=0$ for some $Y \in W\left(r, x_{r, k, a}+1, a\right)$. Take any $X \in W\left(r, x_{r, t, a}, a\right)$. Since $h^{0}\left(\mathcal{O}_{X}(k)\right)=$ $(k+1+a) x_{r, k, a},(1)$ shows that $h^{0}\left(\mathcal{I}_{X}(k)\right)=y_{r, k, a}+h^{1}\left(\mathcal{I}_{X}(k)\right)$.

Fix $P \in \mathbb{P}^{r}, r \geq 2$, and a line $D \subset \mathbb{P}^{r}$. The 2-point $2 P$ is the closed subscheme of $\mathbb{P}^{r}$ with $\left(\mathcal{I}_{P}\right)^{2}$ as its ideal sheaf. The 2-line $2 D$ is the closed subscheme of $\mathbb{P}^{r}$ with $\left(\mathcal{I}_{D}\right)^{2}$ as its ideal sheaf.

Lemma 1. Let $V \subset H^{0}\left(\mathcal{O}_{\mathbb{P}^{r}}(2)\right), r \geq 2$, be a linear system containing a line $L$ in its base locus. Fix a general $P \in L$ and let $v$ be a general tangent vector of $\mathbb{P}^{r}$ with $v_{\text {red }}=\{P\}$. Set $V^{\prime}:=\{f \in V: f \mid v \equiv 0\}$. We have $\operatorname{dim}\left(V^{\prime}\right)=\operatorname{dim}(V)$ if a general $T \in|V|$ is not a quadric cone with $L$ in its vertex and $V^{\prime}=V$ if every $\{g=0\}, g \in V \backslash\{0\}$, is a quadric cone with vertex containing $L$.

Proof. A quadric hypersurface contains every (or a general) degree 2 connected scheme $v$ with $v_{\text {red }}=\{P\}$ if and only if it is a quadric cone with vertex 
containing $P$. A quadric cone has vertex containing $L$ if and only if its vertex contains a general $P \in L$.

Remark 2. Fix positive integers $r, k, t$. Fix any linear subspace of $H^{0}\left(\mathcal{O}_{\mathbb{P}^{r}}(k)\right)$. Let $Z \subset \mathbb{P}^{r}$ be a general union of $t$ tangent vectors. By [8, Lemma 1.4] or [12] (in characteristic zero) or if $\operatorname{char}(\mathbb{K})>k$ ([7], $Z$ imposes $\min \{\operatorname{dim}(V), 2 t\}$ independent conditions to $V$.

\section{Proof in $\mathbb{P}^{3}$}

Let $Q \subset \mathbb{P}^{3}$ be a smooth quadric surface.

Remark 3. No cubic surface $T \subset \mathbb{P}^{3}$ has a singular locus containing 3 disjoint lines (e.g. by Bezout and the fact that 3 disjoint lines of $\mathbb{P}^{3}$ are contained in a smooth quadric surface, but no plane contains two disjoint lines).

Remark 4. We have

$$
\begin{array}{lll}
\left(x_{3,3,0}, y_{3,3,0}\right)=(5,0), & \left(x_{3,3,1}, y_{3,3,1}\right)=(4,0), & \left(x_{3,3,2}, y_{3,3,2}\right)=(3,2), \\
\left(x_{3,3,3}, y_{3,3,3}\right)=(2,6), & \left(x_{3,3,4}, y_{3,3,4}\right)=(2,4), & \left(x_{3,4,0}, y_{3,4,0}\right)=(7,0), \\
\left(x_{3,4,1}, y_{3,4,1}\right)=(5,5), & \left(x_{3,4,2}, y_{3,4,2}\right)=(5,0), & \left(x_{3,4,3}, y_{3,4,3}\right)=(4,3), \\
\left(x_{3,4,4}, y_{3,4,4}\right)=(3,8), & \left(x_{3,4,5}, y_{3,4,5}\right)=(3,5), & \left(x_{3,5,0}, y_{3,5,0}\right)=(9,2), \\
\left(x_{3,5,1}, y_{3,5,1}\right)=(8,0), & \left(x_{3,5,2}, y_{3,5,2}\right)=(7,0), & \left(x_{3,5,3}, y_{3,5,3}\right)=(6,2), \\
\left(x_{3,5,4}, y_{3,5,4}\right)=(5,6), & \left(x_{3,5,5}, y_{3,5,5}\right)=(5,1), & \left(x_{3,5,6}, y_{3,5,6}\right)=(4,7), \\
\left(x_{3,6,0}, y_{3,6,0}\right)=(12,0), & \left(x_{3,6,1}, y_{3,6,1}\right)=(10,4), & \left(x_{3,6,2}, y_{3,6,2}\right)=(9,3), \\
\left(x_{3,6,3}, y_{3,6,3}\right)=(8,4), & \left(x_{3,6,4}, y_{3,6,4}\right)=(7,7), & \left(x_{3,6,5}, y_{3,6,5}\right)=(7,0), \\
\left(x_{3,6,6}, y_{3,6,6}\right)=(6,6), & \left(x_{3,6,7}, y_{3,6,7}\right)=(6,0), & \left(x_{3,7,0}, y_{3,7,0}\right)=(15,0), \\
\left(x_{3,7,1}, y_{3,7,1}\right)=(13,3), & \left(x_{3,7,2}, y_{3,7,2}\right)=(12,0), & \left(x_{3,7,3}, y_{3,7,3}\right)=(10,9), \\
\left(x_{3,7,4}, y_{3,7,4}\right)=(10,0), & \left(x_{3,7,5}, y_{3,7,5}\right)=(9,3), & \left(x_{3,7,6}, y_{3,7,6}\right)=(8,8), \\
\left(x_{3,7,7}, y_{3,7,7}\right)=(8,0), & \left(x_{3,7,8}, y_{3,7,8}\right)=(7,8), & \left(x_{3,8,0}, y_{3,8,0}\right)=(18,3), \\
\left(x_{3,8,1}, y_{3,8,1}\right)=(16,5), & \left(x_{3,8,2}, y_{3,8,2}\right)=(15,0), & \left(x_{3,8,3}, y_{3,8,3}\right)=(13,9), \\
\left(x_{3,8,4}, y_{3,8,4}\right)=(12,9), & \left(x_{3,8,5}, y_{3,8,5}\right)=(11,11), & \left(x_{3,8,6}, y_{3,8,6}\right)=(11,0), \\
\left(x_{3,8,7}, y_{3,8,7}\right)=(10,5), & \left(x_{3,8,8}, y_{3,8,8}\right)=(9,12), & \left(x_{3,8,9}, y_{3,8,9}\right)=(9,3), \\
\left(x_{3,9,0}, y_{3,9,0}\right)=(22,0), & \left(x_{3,9,1}, y_{3,9,1}\right)=(20,0), & \left(x_{3,9,2}, y_{3,9,2}\right)=(18,4), \\
\left(x_{3,9,3}, y_{3,9,3}\right)=(16,12), & \left(x_{3,9,4}, y_{3,9,4}\right)=(15,10), & \left(x_{3,9,5}, y_{3,9,5}\right)=(14,10),
\end{array}
$$




$$
\begin{aligned}
& \left(x_{3,9,6}, y_{3,9,6}\right)=(13,12), \quad\left(x_{3,9,7}, y_{3,9,7}\right)=(12,16), \quad\left(x_{3,9,8}, y_{3,9,8}\right)=(12,4), \\
& \left(x_{3,9,9}, y_{3,9,9}\right)=(11,11), \quad\left(x_{3,9,10}, y_{3,9,10}\right)=(11,0) .
\end{aligned}
$$

Proof of Theorem 1 in $\mathbb{P}^{3}$. By Lemma 1 we may assume $k \geq 3$. Taking the difference between (1) with $\left(r^{\prime}, k^{\prime}, a^{\prime}\right)=(3, k, a)$ and $\left(r^{\prime}, k^{\prime}, a^{\prime}\right)=(3, k-2, a-2)$ we get

$$
\begin{array}{r}
4 x_{3, k-2, a-2}+(k+1+a)\left(x_{3, k, a}-x_{3, k-2, a-2}\right)+y_{3, k, a}-y_{3, k-2, a-2} \\
=(k+1)^{2} .
\end{array}
$$

If $a \geq 3$ (resp. $a \geq 4$ ), then we also have the following relations:

$$
\begin{array}{r}
5 x_{3, k-2, a-3}+(k+1+a)\left(x_{3, k, a}-x_{3, k-2, a-3}\right)+y_{3, k, a}-y_{3, k-2, a-3} \\
=(k+1)^{2}, \\
6 x_{3, k-2, a-4}+(k+1+a)\left(x_{3, k, a}-x_{3, k-2, a-4}\right)+y_{3, k, a}-y_{3, k-2, a-4} \\
=(k+1)^{2}
\end{array}
$$

By [14] and [5] the theorem is true if $a \leq 1$. Fix an integer $a$ with $2 \leq$ $a \leq k+1$. Let $Y \subset \mathbb{P}^{3}$ be a general union of $x_{3, k-2, a-2}(a-2)$-decorated lines. Since $a-2 \leq k-1$, the inductive assumption gives $h^{1}\left(\mathcal{I}_{Y}(k-2)\right)=0$ and $h^{0}\left(\mathcal{I}_{Y}(k-2)\right)=y_{3, k-2, a-2}$. Set $w:=x_{3, k, a}-x_{3, k-2, a-2}$. By (2) we have $w \leq k$ for all $k \geq 3$. We assume $k \geq 5$ and use Lemma 1 and Remark 3 for the integer $k-2$ (plus the explicit values of the integer $x_{3, k-2, a-2}$ ) to handle the cases $k=3,4$. For each $P \in Y \cap Q$ let $v_{P}$ be a general tangent vector of $Q$ with $P$ as its reduction. Set $Z:=\cup_{P \in Y \cap Q} v_{P}$. For general $Y$ the scheme $Z$ is a general union of $x_{3, k-2, a-2}$ tangent vectors of $Q$.

Claim 1. if $2 \leq a \leq k+1$ and either $k \geq 20$ or $a \geq(k+2) / 2$, then $w \leq(k+1) / 2$.

Proof of Claim 1. Assume $w \geq(k+2) / 2$. Since $y_{3, k, a} \geq 0, y_{3, k, a-2} \leq k-3+a$ and $4(k-3+a) x_{3, k-2, a-2}+4 y_{3, k-2, a-2}=4\left(\begin{array}{c}k+1 \\ 3\end{array}\right),(2)$ gives $4\left(\begin{array}{c}k+1 \\ 3\end{array}\right)-(k+1+$ a) $(k-3+a)+(k+1+a)(k-3+a)(k+2) / 2<(k+1+a)(k+1)^{2}$. Hence $4\left(\begin{array}{c}k+1 \\ 3\end{array}\right)<(k+1+a)\left(k^{2}-a k+5 k-4\right) / 2$. Since the right hand side of the last inequality is a decreasing function of $a$ when $a>0$, we get a contradiction for all $k \geq 20$ and, if $a \geq(k+2) / 2$, for all $k \geq 10$. 
(a) In this step we assume $y_{3, k, a} \geq y_{3, k-2, a-2}$. Let $E \subset Q$ be a general union of $w$ lines of type $(0,1)$. For each line $L \subseteq E$ fix a general $S_{L} \subset L$ such that $\sharp\left(S_{L}\right)=a$. For each line $L \subseteq E$ and each $P \in S_{L}$ let $v_{P}$ be a tangent vector of $Q$ with $\left(v_{P}\right)_{\text {red }}=\{P\}$ and not tangent to $L$. Set $S:=\cup_{L \subseteq E} S_{L}$ and $X:=Y \cup Z \cup E \cup \cup_{P \in S} v_{P}$. Since $X$ is a disjoint union of $x_{3, k, a} a$ decorated lines, to prove the theorem for the integers $k$ and $a$ it is sufficient to prove that $h^{1}\left(\mathcal{I}_{X}(k)\right)=0$. Since $\operatorname{Res}_{Q}(X)=Y$, it is sufficient to prove that $h^{1}\left(Q, \mathcal{I}_{X \cap Q}(k)\right)=0$. Since $\operatorname{Res}_{E}(X \cap Q)=S \cup Z$, it is sufficient to prove that $h^{1}\left(Q, \mathcal{I}_{S \cup Z}(k, k-w)\right)=0$. Since $\operatorname{deg}(S \cup Z)=(k+1)(k+1-w)+y_{3, k, a}-$ $y_{3, k-2, a-2}$, it is sufficient to prove that $h^{1}\left(Q, \mathcal{I}_{S}(k, k-w)\right)=0$ (Remark 3). For each line $L \subseteq E$ fix $S_{L}^{\prime} \subset L$ such that $\sharp\left(S_{L}^{\prime}\right)=k+1$ and $S_{L}^{\prime} \supset S_{L}$. It is sufficient to prove that $h^{1}\left(Q, \mathcal{I}_{\cup_{L \subseteq E} S_{L}^{\prime}}(k, k-w)=0\right.$. Assume for the moment that either $k \geq 20$ or $k \geq 10$ and $a \geq(k+2) / 2$. Since $\sharp\left(S_{L}^{\prime}\right)=k+1$ for all $L$, we have $h^{1}\left(Q, \mathcal{I}_{\cup_{L \subseteq E} S_{L}^{\prime}}(k, k-w)=h^{1}\left(Q, \mathcal{O}_{Q}(k, k-2 w)=0\right.\right.$, the latter equality coming from the inequality $2 w \leq k+1$ (Claim 1 ). Now assume $a \leq(k+1) / 2$. In this case we only need the inequality $w+\lceil w / 2\rceil \leq k+1$, instead of the inequality $2 w \leq k+1$; this weaker inequality is true for all $k \geq 5$. The explicit values in Remark 4 show that we alway have $w \leq(k+1) / 2$.

(b) In this step we assume $y_{3, k, a}<y_{3, k-2, a-2}$. Set $e:=y_{3, k-2, a-2}-y_{3, k, a}$. We modify the construction just given in step (a) in the following way. Fix $S^{\prime} \subset Y \cap$ $Q$ such that $\sharp\left(S^{\prime}\right)=e$ and each line of $Y_{\text {red }}$ contains at most one point of $S^{\prime}$; this is possible because $x_{3, k-2, a-2}=\left\lfloor\left(\begin{array}{c}k+1 \\ 3\end{array}\right) /(k-3+a)\right\rfloor \geq k-4+a \geq y_{3, k-2, a-2} \geq e$. Call $R_{1}, \ldots, R_{e}$ the lines of $Y_{\text {red }}$ containing a point of $S^{\prime}$. For each $P \in S^{\prime}$ with, say $P \in R_{i} \cap Q$ let $v_{P}$ be a general tangent vector of $\mathbb{P}^{3}$ with as $v_{\text {red }}$ a general point of $R_{i}$. Set $Y_{1}:=Y \cup \bigcup_{P \in S} v_{P}$. Then take $E \subset Q$ and $v_{P} \subset Q, P \in S_{L} \subset L$, $L \subseteq E$ as in step (a). Set $X:=Y_{1} \cup E \cup \bigcup_{P \in S_{L}, L \subseteq E} v_{P}$. It is sufficient to prove that $h^{1}\left(\mathcal{I}_{X}(k)\right)=0$. As in step (a) we have $h^{1}\left(Q, \mathcal{I}_{X \cap Q}(k)\right)=0$. Hence it is sufficient to prove that $h^{1}\left(\mathcal{I}_{\operatorname{Res}_{Q}(X)}(k-2)\right)=0$. We have $\operatorname{Res}_{Q}(X)=Y_{1}$. Assume $f:=h^{1}\left(\mathcal{I}_{Y_{1}}(k-2)\right)>0$. Since $Y \subset Y_{1}, h^{1}\left(\mathcal{I}_{Y}(k-2)\right)=0$ and the ideal sheaf of $Y$ in $Y_{1}$ is a skyscraper sheaf with $S$ as its support and length 1 at each $P \in S$, we have $e \leq \sharp\left(S^{\prime}\right)=e$ and we may order the lines $L_{1}, \ldots, L_{x_{3, k-2, a-2}}$ of $Y_{\text {red }}$ so that $S^{\prime} \subset\left(L_{1} \cup \cdots \cup L_{y_{3, k-2, a-2}-y_{3, k, a}}\right) \cap Q$ and, calling $Y[i], 1 \leq i \leq y_{3, k-2, a-2}-y_{3, k, a}$, the union of $Y$ and the schemes $v_{P}$, $P \in Q \cap\left(L_{1} \cup \cdots \cup L_{i}\right)$ (with $Y[0]:=Y$ ), we have $h^{1}\left(\mathcal{I}_{Y[i]}(k-2)\right)=0$ for all $i \leq \alpha:=w-f$ and $h^{0}\left(\mathcal{I}_{Y[\alpha]}(k-2)\right)=h^{0}\left(\mathcal{I}_{Y[\alpha+1}(k-2)\right)$. Call $D_{1}, \ldots, D_{\alpha}$ the lines of $Y_{\text {red }}$ supporting the ideal sheaf of $Y$ in $Y[\alpha]$ and $D_{\alpha+1}, \ldots D_{y_{3, k-2, a-2}}$ all the other lines of $Y_{\text {red }}$. Moving $D_{\alpha+1}, \ldots D_{y_{3, k-2, a-2}}$ and the vectors $v_{P}$ at all points of the lines $R_{i}$ not on some $D_{1}, \ldots, D_{\alpha}$, we get a monodromy group which is a full symmetric group. Hence every surface $T \in\left|\mathcal{I}_{Y[\alpha]}(k-2)\right|$ contains 
the double line $D_{i}$. Call $W$ the union of these double lines and of the connected components of $Y_{1}$ with $D_{1}, \ldots, D_{\alpha}$ as its support. We have $h^{0}\left(\mathcal{I}_{Y_{1}}(k-2)\right)=$ $h^{0}\left(\mathcal{I}_{W}(k-2)\right)=y_{3, k-2, a-2}-\alpha$ and $W \cap Q$ is a general union of $\alpha$ tangent vectors of $Q, \alpha$ points of $Q$ and $2\left(x_{3, k-2, a-2}-\alpha\right) 2$-points of $Q$. Let $E_{1} \subset Q$ be a general union of $w 1$ lines of type $(0,1)$. For each $L \subseteq E_{1}$ take subset $S_{L} \subset L$ with $\sharp\left(S_{L}\right)=k+1$. For any line $L \subseteq E_{1}$ and any $P \in S_{L}$ let $v_{P}$ be any tangent vector of $Q$ with $P$ as its reduction and not contained in $L$. Let $L \subset Q$ be a general line of type $(1,0)$. Set $X_{1}:=Y_{1} \cup L \cup E_{1} \cup \bigcup_{P \in S_{L}, L \subseteq E_{1}} v_{P}$. Since $X_{1}$ is a disjoint union of $x_{3, k, a}-1 a$-decorated lines and $y_{3, k-2, a-2}-\alpha<2 k+1+y_{3, k, a}$, to get a contradiction it is sufficient to prove that $h^{0}\left(\mathcal{I}_{X_{1}}(k)\right) \leq y_{3, k-2, a-2}-\alpha$. Since $h^{0}\left(\mathcal{I}_{Y_{1}}(k-2)\right)=h^{0}\left(\mathcal{I}_{W}(k-2)\right)=y_{3, k-2, a-2}-\alpha$, it is sufficient to prove that $h^{0}\left(Q, \mathcal{I}_{Q \cap W}(k, k+1-w)\right)=0$. This is true by (2) and [15], because $\operatorname{deg}(W \cap Q)-\operatorname{deg}(Y(Y \cup Z) \cap Q))>k+1+e$.

(c) To check that $h^{0}\left(\mathcal{I}_{U}(k)\right)=0$ for some $U \in W\left(r, x_{3, k, a}+1, a\right)$ modify step (b) taking $e:=y_{3, k-2, a-2}$ and adding one more $a$-line in $Q$.

\section{Proof in $\mathbb{P}^{r}, r \geq 4$}

We fix an integer $r \geq 4$ and we assume that Theorem 1 is true in $\mathbb{P}^{r-1}$. By [5] to prove Theorem 1 in $\mathbb{P}^{r}$ we may assume $a \geq 2$. We have $a \leq k+1$ by assumption. Since Theorem 1 is true for the integer $k=2$ (Lemma 1 ) we may assume $k \geq 3$ and that Theorem 1 is true for the triple $\left(r^{\prime}, k^{\prime}, a^{\prime}\right)=(r, k-1, a-1)$. Let $H \subset \mathbb{P}^{r}$ be a hyperplane. Taking the difference between (1) and the same equation for the integers $r, a^{\prime}:=a-1$ and $k^{\prime}:=k-1$ we get

$$
\begin{array}{r}
2 x_{r, k-1, a-1}+(k+1+a)\left(x_{r, k, a}-x_{r, k-1, a-1}\right)+y_{r, k, a}-y_{r, k-1, a-1} \\
=\left(\begin{array}{c}
r+k-1 \\
r-1
\end{array}\right) .
\end{array}
$$

Set $w:=x_{r, k, a}-x_{r, k-1, a-1}$.

Claim 1. We have $w \geq 0$.

Proof of Claim 1. Assume $w \leq-1$. Since $x_{r, k-1, a-1}=\left\lfloor\left(\begin{array}{c}r+k-1 \\ r\end{array}\right) /(k+a-1)\right\rfloor$, $y_{r, k, a} \leq k+a$ and $y_{r, k-1, a-1} \geq 0$, (5) gives the inequality $2\left(\begin{array}{c}r+k-1 \\ r\end{array}\right)-2(k+a-1) \geq$ $(k+a-1)\left(\begin{array}{c}r+k-1 \\ r-1\end{array}\right)$. Since $a>0$ and $k\left(\begin{array}{c}r+k-1 \\ r-1\end{array}\right)-2\left(\begin{array}{c}r+k-1 \\ r\end{array}\right)=\left(\begin{array}{c}r+k-1 \\ r\end{array}\right)[r-2]>0$, we get a contradiction.

Let $Y \subset \mathbb{P}^{r}$ be a general union of $x_{r, k-1, a-1}(a-1)$-decorated lines. By the inductive assumption we have $h^{0}\left(\mathcal{I}_{Y}(k-1)\right)=0$ and $h^{0}\left(\mathcal{I}_{Y}(k-1)\right)=y_{r, k-1, a-1}$. 
Since $Y$ is general $Y \cap H$ is a general subset of $H$ with cardinality $x_{r, k-1, a-1}$. For each $P \in H \cap Y$ let $v_{P}$ be a general tangent of $H$ with $\{P\}=\left(v_{P}\right)_{\text {red }}$. The scheme $Y \cup Z$ is a disjoint union of $x_{r, k-1, a-1}(a-1)$-decorated lines, $\operatorname{Res}_{H}(Y \cup Z)=Y$ and $(Y \cup Z) \cap H=Z$ (scheme-theoretic intersection). We have $\operatorname{deg}(Z)=2 x_{r, k-1, a-1}$.

(a) In this step we assume $y_{r, k, a} \geq y_{r, k, a-1}$. Let $E \subset H$ be a general union of $w a$-decorated lines of $H$. Set $X:=Y \cup Z \cup E$. Since $\operatorname{Res}_{H}(X)=Y$, $X \cap H=Z \cup E$ and $h^{1}\left(\mathcal{I}_{Y}(k-1)\right)=0$, a Castelnuovo's sequence shows that it is sufficient to prove that $h^{1}\left(H, \mathcal{I}_{Z \cup E}(k)\right)=0$. By $(5)$ we have $h^{0}\left(\mathcal{O}_{X \cup E}(k)\right)=$ $\left(\begin{array}{c}r+k-1 \\ r-1\end{array}\right)+y_{r, k-1, a-1}-y_{r, k, a} \leq\left(\begin{array}{c}r+k-1 \\ r-1\end{array}\right)$. By Remark 3 it is sufficient to prove that $h^{1}\left(H, \mathcal{I}_{E}(k)\right)=0$. This vanishing is true, because $H \cong \mathbb{P}^{r-1}$.

(b) In this step we assume $y_{r, k, a}<y_{r, k, a-1}$ and $w \geq y:=y_{r, k, a-1}-y_{r, k, a}$. Set $a^{\prime}:=\min \{a-1, k-1\}$ and let $G \subseteq Y$ be any union of $\left(a^{\prime}\right)$-decorated lines. Since $h^{0}\left(\mathcal{I}_{Y}(k-1)\right)=y_{r, k-1, a-1} \leq k-2-a<\left(\begin{array}{c}r+k-1 \\ r\end{array}\right)-\left(\begin{array}{c}r+k-2 \\ r\end{array}\right)$, the inductive assumption gives $h^{0}\left(\mathcal{I}_{G}(k-2)\right)=0$. Hence $h^{0}\left(\mathcal{I}_{Y}(k-1)\right)=0$ (this vanishing is easily proved, without using induction on $k$ ). Let $F \subset H$ be a general union of $w$ lines. Write $F=A \sqcup B$ with $B$ union of $y$ lines and $A$ union of $w-y$ lines. Let $A^{\prime} \subset H$ be a general union of $a$-decorated lines of $H$ with $A_{\text {red }}^{\prime}=A^{\prime}$. Let $B^{\prime \prime} \subset \mathbb{P}^{r}$ be a general union of $a$-decorated lines with the following restriction; for each line $D \subseteq B$ let $E_{D}$ be the connected component of $B^{\prime \prime}$ with $D$ as its support; we assume that $E_{D} \cap H$ is an $(a-1)$-decorated lines and hence the scheme $\operatorname{Res}_{H}\left(E_{D}\right)$ is a point of $D$. Set $X:=Y \cup \bigcup Z \cup A^{\prime} \cup B^{\prime \prime}$ and $S^{\prime}:=\operatorname{Res}_{H}\left(B^{\prime \prime}\right)$. We have $\operatorname{Res}_{H}(X)=Y \cup S^{\prime}$. Since $B$ is a general union of $y$ lines of $H$ and each point $\operatorname{Res}_{H}\left(E_{D}\right), D$ a connected component of $B$, is a general point of $D, S^{\prime}$ is a general union of $y$ points of $H$. Since $\operatorname{Res}_{H}(Y)=Y$, $S^{\prime}$ is general in $H$ and $h^{0}\left(\mathcal{I}_{Y}(k-2)\right)=0$, we have $h^{0}\left(\mathcal{I}_{Y \cup S^{\prime}}(k-1)\right)=y_{r, k, a}$ and $h^{1}\left(\mathcal{I}_{Y \cup S^{\prime}}(k-1)\right)=0$ (see, e.g., [6], Lemma 4). A Castelnuovo's sequence gives $h^{1}\left(\mathcal{I}_{X}(k)\right)=0$.

(c) In this step we assume $y_{r, k, a}<y_{r, k, a-1}$ and $w<y:=y_{r, k, a-1}-y_{r, k, a}$. We have $x_{r, k-1, a-1}=\left\lfloor\left(\begin{array}{c}r+k-1 \\ r\end{array}\right) /(k-1-a)\right\rfloor>2 y$

Claim 2. We have $x_{r, k-1, a-1}-y>\left(\begin{array}{c}t+k-1 \\ r-1\end{array}\right) / r$.

Proof of Claim 2. By (5) and the assumption $w<y:=y_{r, k, a-1}-y_{r, k, a}$ we have $2 x_{r, k-1, a-1}-2 y+(k+1+a) y-k-1-a \geq\left(\begin{array}{c}r+k-1 \\ r-1\end{array}\right)+y$. Since $r \geq 4$ and $x_{r, k-1, a-1}=\left\lfloor\left(\begin{array}{c}r+k-1 \\ r\end{array}\right) /(k-1-a)\right\rfloor>2 y$, we get $r\left(x_{r, k-1, a-1}-y\right)>\left(\begin{array}{c}t+k-1 \\ r-1\end{array}\right)$. For each $P \in Y \cap H$ let $D_{P}$ be the line of $Y_{\text {red }}$ containing $P$. Fix $S \subset Y \cap H$ such that $\sharp(S)=y$. Set $Z^{\prime}:=\cup_{P \in Y \cap H \backslash S} v_{P}$. Let $Y^{\prime}$ be the union of the connected 
components of $Y$ with $D_{P}, P \in Y \cap H \backslash S$ as their support. $Y^{\prime}$ is a general union of $x_{r, k-1, a-1}-y_{r, k, a-1}+y_{r, k, a}(a-1)$-decorated lines. For each $P \in S$ fix a general $O_{P} \in D_{P}$ and let $w_{P}$ be a general tangent vector of $\mathbb{P}^{r}$ with $O_{P}$ as its reduction. The scheme $Y_{1}:=Y \cup \cup_{P \in S} w_{P}$ is a general union of $x_{r, k-1, a-1}-y(a-1)$-decorated lines and $y a$-decorated lines. Set $Y_{2}:=Y_{1} \cup Z^{\prime}$. $Y_{2}$ is a disjoint union of $x_{r, k-1, a-1} a$-decorated lines, $Y \cap Y_{2}=Z^{\prime} \cup S$ and $\operatorname{Res}_{H}\left(Y_{2}\right)=Y_{1}$. We have $h^{0}\left(\mathcal{I}_{Y_{1}}(k-1)\right)=y_{r, k, a}+h^{1}\left(\mathcal{I}_{Y_{1}}(k-1)\right)$. First assume $h^{1}\left(\mathcal{I}_{Y_{1}}(k-1)\right)=0$. In this case to prove Theorem 1 for the triple $(r, k, a)$ it is sufficient to repeat the proof of step (a) adding to $Y_{2}$ a general union of $w a$-decorated lines of $H$. Now assume $h^{1}\left(\mathcal{I}_{Y_{1}}(k-1)\right)>0$. Since $h^{1}\left(\mathcal{I}_{Y}(k-1)\right)=0$, we may order the points of $S$, say $S=\left\{P_{1}, \ldots, P_{y}\right\}$, so that the $h^{1}\left(\mathcal{I}_{Y \cup \bigcup_{1 \leq i \leq e} w_{P}}(k-1)\right)=0$ and $h^{1}\left(\mathcal{I}_{Y \cup \bigcup_{1 \leq i \leq e+1}} w_{P_{i}}(k-1)\right)>0$. Since $w_{P_{e+1}}$ is a general tangent vector of $\mathbb{P}^{r}$ supported by a general point of $D_{P_{e+1}}$, we get that every $T \in\left|\mathcal{I}_{Y \cup \bigcup_{1<i<e+1} w_{P_{i}}}(k-1)\right|$ contains the double line $2 D_{P_{e+1}}$ of $\mathbb{P}^{r}$. The monodromy group of the symmetric product of $x_{r, k-1, a-1}-e$ copies of $H$ is the full symmetric group and for each $P \in H$ the set of all $(a-1)$-decorated lines of $\mathbb{P}^{r}$ containing $P$, but intersecting $H$ only at $P$ is irreducible. Hence a monodromy argument gives that every $T \in\left|\mathcal{I}_{Y \cup \bigcup_{1 \leq i \leq e} w_{P_{i}}}(k-1)\right|$ contains the 2-line $2 D_{P}$ for all $P \in Y \cap H \backslash S$. Fix a general hyperplane $M \subset \mathbb{P}^{r}$. For general $Y$ the scheme $T \cap M$ contains $x_{r, k-1, a-1}-y$ general 2-points; call $W \subset M$ the union of these 2-points. Assume for the moment either $k \geq 4$ and $r \geq 6$ or $r=4,5$ and $k \geq 5$. By Claim 2 and a theorem of Alexander-Hirschowitz ([1], [2], [3], [4], [13]), we have $h^{0}\left(M, \mathcal{I}_{W}(k-1)\right)=0$. Hence $M \subset T$. Varying $M$ we get a contradiction (it is even easily proved that no $T^{\prime} \in\left|\mathcal{I}_{Y}(k-1)\right|$ contains a hyperplane). Now assume $k=3$. In this case we get $h^{0}\left(\mathcal{I}_{Y \cup \cup_{1 \leq i \leq e} w_{P}}(2)\right)=0$, because $Y_{\text {red }}$ spans $\mathbb{P}^{r}$ and the singular locus of a quadric hypersurface is a linear subspace. In the case $k=4$ and $r=4$ use Remark 2. Now assume $k=4$ and $r=5$. We have $r_{5,3, a-1} \geq\left\lfloor\left(\begin{array}{l}8 \\ 3\end{array}\right) / 8\right\rfloor=7$ and no cubic hypersurface of $\mathbb{P}^{4}$ has a singular locus containing 7 general lines (e.g., use a Castelnuovo's sequence).

(d) A small adaptation of steps (b) and (c) gives that $h^{0}\left(\mathcal{I}_{A}(k)\right)=0$ for some $A \in W\left(r, x_{r, k, a}+1, a\right)$.

Remark 5. To prove an equivalent of Theorem 1 for larger values of $a$, say $k+2 \leq a \leq 2 k$, one should use (3) and 4). Fix $Y \in W\left(3, x_{3, k-2, a-2}, a-2\right)$ as in the proof of the case $r=3$ of Theorem 1 as in the proof and a line $D \subset Y_{\text {red }}$. Notice that the 2-point of $Q$ with $P$ as its support is the flat limit of a family of tangent vectors of $\mathbb{P}^{3}$ supported by general points of $D$. The same observation may be used in $\mathbb{P}^{r}$, using both Alexander-Hirschowitz theorem and [9]. 


\section{Acknowledgements}

The author was partially supported by MIUR and GNSAGA of INdAM (Italy).

\section{References}

[1] J. Alexander, Singularités imposables en position générale aux hypersurfaces de $\mathbb{P}^{n}$, Compositio Math. 68 (1988), 305-354.

[2] J. Alexander and A. Hirschowitz, Un lemme d'Horace différentiel: application aux singularité hyperquartiques de $\mathbb{P}^{5}$, J. Alg. Geom. 1 (1992), $411-426$.

[3] J. Alexander and A. Hirschowitz, La méthode d'Horace éclaté: application à l'interpolation en degré quatre, Invent. Math. 107 (1992), 585-602.

[4] J. Alexander and A. Hirschowitz, Polynomial interpolation in several variables, J. Alg. Geom. 4 (1995), 201-222.

[5] E. Ballico, Postulation of general unions of lines and decorated lines, preprint.

[6] E. Ballico, Postulation of general unions of lines and multiplicity two points in $\mathbb{P}^{r}, r \leq 5$, preprint.

[7] E. Ballico, Postulation of unions if tangent vectors, preprint.

[8] A. Bernardi, M. V. Catalisano, A. Gimigliano and M. Idà, Secant varieties to osculating varieties of Veronese embeddings of $\mathbb{P}^{n}$. J. Algebra 321 (2009), no. $3,982-1004$.

[9] M. C. Brambilla and G. Ottaviani, On partial polynomial interpolation. Linear Algebra Appl. 435 (2011), no. 6, 1415-1445.

[10] E. Carlini, M. V. Catalisano and A. V. Geramita, 3-dimensional sundials, Cent. Eur. J. Math. 9 (2011), no. 5, 949-971.

[11] E. Carlini, M. V. Catalisano and A. V. Geramita, Reduced and nonreduced linear spaces: lines and points, arXiv: 1308.0796.

[12] C. Ciliberto and R. Miranda, Interpolations on curvilinear schemes, J. Algebra 203 (1998), no. 2, 677-678. 
[13] K. Chandler, A brief proof of a maximal rank theorem for generic 2-points in projective space. Trans. Amer. Math. Soc. 353 (2000), no. 5, 1907-1920.

[14] R. Hartshorne and A. Hirschowitz, Droites en position générale dans $\mathbb{P}^{n}$, Algebraic Geometry, Proceedings, La Rábida 1981, 169-188, Lect. Notes in Math. 961, Springer, Berlin, 1982.

[15] A. Laface, On linear systems of curves on rational scrolls, Geom. Dedicata 90 (2002), 127-144. 